\title{
A new locking-free formulation for planar, shear deformable, linear and quadratic beam finite elements based on the absolute nodal coordinate formulation
}

\author{
Karin Nachbagauer • Astrid S. Pechstein • \\ Hans Irschik · Johannes Gerstmayr
}

Received: 15 October 2010 / Accepted: 8 February 2011 / Published online: 8 April 2011

(C) The Author(s) 2011. This article is published with open access at Springerlink.com

\begin{abstract}
Many widely used beam finite element formulations are based either on Reissner's classical nonlinear rod theory or the absolute nodal coordinate formulation (ANCF). Advantages of the second method have been pointed out by several authors; among the benefits are the constant mass matrix of ANCF elements, the isoparametric approach and the existence of a consistent displacement field along the whole cross section. Consistency of the displacement field allows simpler, alternative formulations for contact problems or inelastic materials. Despite conceptional differences of the two formulations, the two models are unified in the present paper.

In many applications, a nonlinear large deformation beam element with bending, axial and shear deformation properties is needed. In the present paper, linear and quadratic ANCF shear deformable beam finite elements are presented. A new locking-free continuum mechanics based formulation is compared to the classical Simo and Vu-Quoc formulation based on Reissner's virtual work of internal forces. Additionally, the introduced linear and quadratic ANCF elements are compared to a fully parameterized ANCF element from the literature. The performance of the respective elements is evaluated through analysis of conventional static and dynamic example problems. The investigation shows that the obtained linear and quadratic ANCF elements are advantageous compared to the original fully parameterized ANCF element.
\end{abstract}

K. Nachbagauer $(\varangle) \cdot$ A.S. Pechstein · H. Irschik

Institute of Technical Mechanics, Johannes Kepler University Linz, Altenbergerstraße 69, 4040 Linz, Austria

e-mail: karin.nachbagauer@jku.at

A.S. Pechstein

e-mail: astrid.pechstein@jku.at

H. Irschik

e-mail: hans.irschik@jku.at

J. Gerstmayr

Linz Center of Mechatronics GmbH, Altenbergerstraße 69, 4040 Linz, Austria

e-mail: johannes.gerstmayr@lcm.at 
Keywords Shear deformable beam · Timoshenko beam · Finite elements · Classical nonlinear rod formulation - Absolute nodal coordinate formulation

\section{Introduction}

Many of the currently used large deformation beam elements are based on two different theories: Reissner's nonlinear rod theory [11] and the absolute nodal coordinate formulation (ANCF) by Yakoub and Shabana [16]. Finite elements based on Reissner's theory have been presented first by Simo and Vu-Quoc in [14]. The absolute nodal coordinate formulation [13] was developed as an alternative for the modeling of large deformation structural problems in two and three space dimensions. The main conceptual difference in contrast to classical large rotation vector formulation is the absence of rotational degrees of freedom. In a recent study [2], it has been shown that Bernoulli-Euler ANCF elements may differ from classical beam theories such as the Euler Elastica, and it has been demonstrated how the results of both theories can be brought into agreement. In a further work [3], it has been shown that Reissner's shear deformable beam, as implemented by Simo and Vu-Quoc [14], can also be implemented with ANCF elements such that the static and dynamic behaviors are fully equivalent. However, in [3] the order of interpolation of axial position and cross section orientation was not optimal and different from the approach of Simo and Vu-Quoc [14].

In contrast to formulations in which displacements and rotations [14] or displacements and slopes [12] are interpolated, different formulations exist, which employ strains as basic interpolated variables and therefore do not suffer from locking; see e.g. [1]. For further information on various types of locking, such as Poisson locking, see [15], or combined shear and thickness locking, see [4]. In the present paper, the ANCF and the classical nonlinear rod theory are unified in order to describe a linear and a quadratic shear deformable beam element; see Fig. 1. In contrast to earlier works on shear deformable ANCF elements, e.g. [7-9], the strain energy is computed according to Simo and Vu-Quoc [14]. For the interpolation of displacements and displacement gradients in the linear ANCF element, linear shape functions are chosen according to [8]. In the quadratic ANCF element, quadratic shape functions similar to [9] are used for the interpolation of displacements and displacement gradients. The kinematic description of the elements is chosen in a way similar to [7], while the strain energy is considerably different from [7]. Here, a detailed description and convergence analysis is presented and compared to the standard ANCF element proposed in [3], which is originally based on the large rotation vector formulation by Simo and Vu-Quoc [14]. In the sequel, this element is referred to as fully parameterized ANCF element [3]. The position vector of the fully parameterized ANCF element [3] uses third-order interpolation, while the rotation of the cross section is only interpolated linearly with respect to the beam axis.

Sections 2 and 3 cover the geometric description of the finite elements, as well as the choice of degrees of freedom for the linear and quadratic ANCF elements. Section 4 deals with different definitions of strain energy for the ANCF beam elements. The present work

Fig. 1 Position vector and slopes of the planar shear deformable absolute nodal coordinate beam finite element: (a) linear element, (b) quadratic element

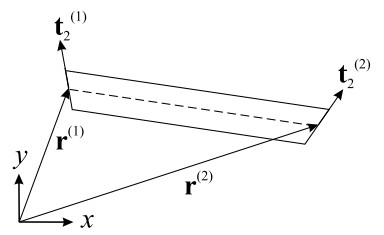

(a)



(b) 
covers a formulation of the elastic forces based on Reissner's nonlinear rod theory, as well as a continuum mechanics approach based on a St. Venant-Kirchhoff material law. For a continuum mechanics based derivation of Reissner's finite-strain beam theory, see [5]. Note that it is possible to transform any suitable continuum mechanics based constitutive law from the continuum level to the beam level; for details, refer to Irschik and Gerstmayr [6].

In the original shear deformable ANCF element by Omar and Shabana [10], the strain energy is defined with a St. Venant-Kirchhoff material law, which uses a linear relation between the Green strain tensor and the second Piola-Kirchhoff stress tensor. In the sequel, the latter formulation is referred to as standard continuum mechanics based formulation, and it is presented in Sect. 4.1. The main problem of the formulation introduced in [10] is the Poisson locking phenomenon.

To avoid the locking effect, the strain energy is modified. To be more precise, the elasticity tensor is split into two parts. The first part does not take into account the Poisson ratio $v$, while the second part covers the Poisson effect; for details, refer to Sect. 4.2. This adapted formulation is denoted as enhanced continuum mechanics based formulation.

The definition of strain energy based on Reissner's nonlinear rod theory is presented in Sect. 4.3. The generalized strains in this formulation can be related to the deformation field of the ANCF element, see [3]. As proposed by Simo and Vu-Quoc [14], shear locking is eliminated by means of reduced integration. The special choice of degrees of freedom allows thickness deformation of the proposed elements. The strain energy is enhanced by a corresponding term, which is not present in the original strain energy by Simo and VuQuoc [14]. The thickness deformation of the beams is of interest e.g. for applications such as rolling mills. The according linear and quadratic finite elements are investigated using several static and dynamic numerical examples. Results are presented in Sect. 5.

The main result of the present paper is that the strain energy of the proposed ANCF elements is equivalent to that of the fully parameterized ANCF element [3]. Moreover, the proposed linear ANCF element has less degrees of freedom, but shows the same convergence order, as the fully parameterized ANCF element [3]. The proposed quadratic ANCF element has the same number of degrees of freedom as the fully parameterized ANCF element [3] but with a higher order of convergence.

\section{Geometric description of the finite elements}

The aim of this section is the definition of the geometric setup of a shear deformable beam finite element. The shear deformation can be taken into account by means of an additional angle between the beam axis and the normal to the cross section. In the paper by Simo and Vu-Quoc [14], a deformation angle was suggested to parameterize the cross-sectional deformation. On the contrary, Omar and Shabana [10] designed an ANCF element, in which slope vectors are used. For the present element, the latter approach is used; however, only one slope vector is employed to model the deformation of the cross section. More precisely, the transverse gradient vector or slope vector, which points in direction of the cross section, is utilized similarly to [7]. The element is thus parameterized by displacements and displacement gradients at specific nodes which form the degrees of freedom. The position vector of the element nodes specifies the position of the beam axis, while the direction of the transverse gradient models shear deformation, whereas its length represents thickness deformation.

Due to the fact that only parts of the deformation gradient are considered, the element is not fully parameterized. An example for a fully parameterized element is [3]. Nevertheless, the nodal coordinates can be related to the nodal coordinates used by Simo and 
Fig. 2 Different configurations of the beam: (a) unit element, (b) straight scaled reference element in unit configuration, (c) reference element and (d) deformed element in deformed configuration

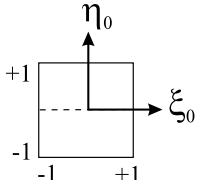

(a)

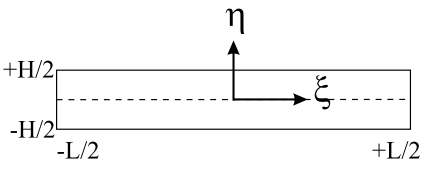

(b)

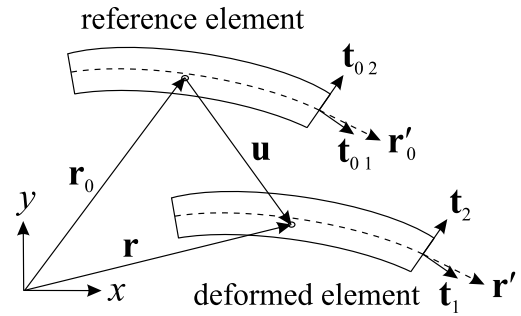

(c)

Vu-Quoc [14], not taking into account thickness deformation. Since the interpolation of the geometry in the reference configuration and the displacements will be based on a single set of functions, the finite element approach is called isoparametric. Four different configurations of the element, depicted in Fig. 2, are considered:

(a) the unit element ranging on the unit square $[-1,1] \times[-1,1]$, which is embedded in the local dimensionless coordinate system $\left(\xi_{0}, \eta_{0}\right)$

(b) the scaled straight element of length $L$ and height $H$, measured in meters, which is embedded in the local coordinate system $(\xi, \eta)$, and which allows the definition of slope vectors

(c) the reference element, which represents the stress-free, possibly distorted configuration of the element in the global coordinate system $(x, y)$

(d) the deformed element in the global coordinate system $(x, y)$

The transformation from the unit element (a) to the scaled straight element (b) leads to a scaling of coordinates

$$
\xi=\frac{L \xi_{0}}{2} \quad \text { and } \quad \eta=\frac{H \eta_{0}}{2} .
$$

It is essential to define the slope vectors as derivatives with respect to the local coordinate system $(\xi, \eta)$ and not the dimensionless coordinates $\left(\xi_{0}, \eta_{0}\right)$, since otherwise the coordinate transformation (1) would lead to additional scaling factors for the slope vectors. Therefore the shape functions will be defined for the scaled straight element (b), i.e. as functions of $\xi$ and $\eta$. The configurations (c) and (d) could also be described in coordinate-free notation. However, for the computer implementation, a certain global coordinate system $(x, y)$ is chosen. All quantities are represented in this global coordinate system $(x, y)$.

The position vector of the local point $(\xi, \eta)$ in the undeformed geometry shall be denoted by $\mathbf{r}_{0}(\xi, \eta)$. It corresponds to the position vector $\mathbf{r}(\xi, \eta)$ in the deformed geometry, see Fig. 2. Introducing the displacement vector $\mathbf{u}(\xi, \eta)$ yields

$$
\mathbf{r}(\xi, \eta)=\mathbf{r}_{0}(\xi, \eta)+\mathbf{u}(\xi, \eta)
$$

Throughout the following, primes indicate the derivative with respect to the local axial coordinate $\xi, \mathbf{r}^{\prime}=\frac{\partial \mathbf{r}}{\partial \xi}$. For the description of the slope vectors of the beam cross section, the dis- 
placement gradients with respect to the transverse coordinate $\eta$ are used, which are denoted by $\mathbf{r}_{, \eta}=\frac{\partial \mathbf{r}}{\partial \eta}$. The position vector of the axis $\mathbf{r}$ is defined as

$$
\mathbf{r}(\xi)=\mathbf{r}(\xi, 0) \text {. }
$$

Then, $\mathbf{r}^{\prime}$ is the tangent direction of the beam centerline. The vector $\mathbf{t}_{2}$ is defined to point in direction of the deformed beam cross section, while $\mathbf{t}_{1}$ is perpendicular to $\mathbf{t}_{2}$. All quantities associated with the reference configuration are indexed with subscript zero, e.g. $\mathbf{t}_{01}, \mathbf{t}_{02}$ and $\mathbf{r}_{0}^{\prime}$ in the reference configuration correspond to $\mathbf{t}_{1}, \mathbf{t}_{2}$ and $\mathbf{r}^{\prime}$ in deformed configuration.

\section{A linear and quadratic ANCF elements}

In the following, both a linear and a quadratic ANCF elements based on the geometrical description from Sect. 2 shall be defined. The degrees of freedom are defined in two and three nodes, respectively. In contrast to standard ANCF elements, in which position and slope vectors are chosen, the respective displacement-based quantities, i.e. the nodal displacements and change of slope vectors, are used here. Appropriate linear and quadratic shape functions are defined.

\subsection{Linear ANCF element}

For the linear ANCF beam element, two nodes are chosen at the ends of the centerline of the beam. A sketch of the element is provided in Fig. 1(a). Four element coordinates are defined in each node $n$, namely the components of the nodal displacement $\mathbf{u}^{(n)}$ and $\mathbf{u}_{, \eta}^{(n)}$, the change of the slope vector,

$$
\mathbf{q}^{(n)}=\left[\begin{array}{ll}
\mathbf{u}^{(n) T} & \mathbf{u}_{, \eta}^{(n) T}
\end{array}\right]^{T}=\left[\begin{array}{llll}
u_{x}^{(n)} & u_{y}^{(n)} & u_{x, \eta}^{(n)} & u_{y, \eta}^{(n)}
\end{array}\right]^{T} .
$$

The displacements $\mathbf{u}^{(1)}, \mathbf{u}^{(2)}$ and displacement gradients $\mathbf{u}_{, \eta}^{(1)}, \mathbf{u}_{, \eta}^{(2)}$ at the nodal points are chosen as element degrees of freedom, leading to the vector of unknowns:

$$
\mathbf{q}=\left[\begin{array}{lll}
\mathbf{q}^{(1) T} & \mathbf{q}^{(2) T}
\end{array}\right]^{T}=\left[\begin{array}{llll}
\mathbf{u}^{(1) T} & \mathbf{u}_{, \eta}^{(1) T} & \mathbf{u}^{(2) T} & \mathbf{u}_{, \eta}^{(2) T}
\end{array}\right]^{T} .
$$

The reference element in the linear case is determined by the generalized coordinate vector


be seen in Fig. 3. The displacement vector $\mathbf{u}$ is interpolated linearly not only on the beam centerline, but on the whole element. It is given in terms of the $m=4$ shape functions $S_{i}$ and the $2 m$ element coordinates $q_{j}$ listed in the vector of unknowns $\mathbf{q}=\left[q_{1}, \ldots, q_{8}\right]^{T}$ :

$$
\mathbf{u}(\xi, \eta)=\sum_{i=1}^{m} S_{i}(\xi, \eta)\left[\begin{array}{ll}
q_{2 i-1} & q_{2 i}
\end{array}\right]^{T} .
$$

In order to obtain sparse mass and stiffness matrices, the shape functions have to be chosen such that they vanish in all but one degree of freedom. This leads to four scalar boundary conditions, which are sufficient to define the linear shape functions uniquely. According to [8] they read

$$
\begin{array}{ll}
S_{1}(\xi, \eta)=\frac{1}{L}\left(\frac{L}{2}-\xi\right), & S_{2}(\xi, \eta)=\eta S_{1}(\xi, \eta), \\
S_{3}(\xi, \eta)=\frac{1}{L}\left(\frac{L}{2}+\xi\right), & S_{4}(\xi, \eta)=\eta S_{3}(\xi, \eta) .
\end{array}
$$


Fig. 3 Configuration of the linear undeformed reference element, in which $q_{0 j}$ are the entries of the generalized coordinate vector $\mathbf{q}_{0}$



Fig. 4 Configuration of the quadratic undeformed reference element, in which $q_{0 j}$ are the entries of the generalized coordinate vector $\mathbf{q}_{0}$

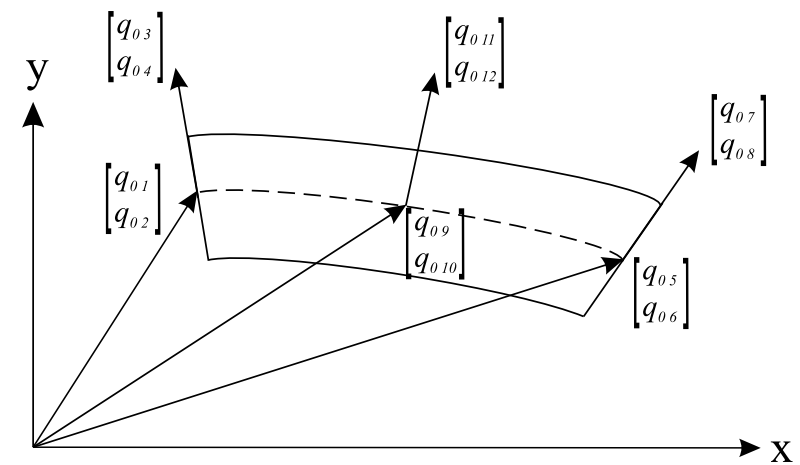

The shape function matrix is built from the shape functions in the following way:

$$
\mathbf{S}=\left[\begin{array}{cccccccc}
S_{1} & 0 & S_{2} & 0 & S_{3} & 0 & S_{4} & 0 \\
0 & S_{1} & 0 & S_{2} & 0 & S_{3} & 0 & S_{4}
\end{array}\right]
$$

Equation (2) now can be rewritten in terms of the shape function matrix $\mathbf{S}$ and the generalized coordinate vectors $\mathbf{q}$ and $\mathbf{q}_{0}$ :

$$
\mathbf{r}=\mathbf{S}\left(\mathbf{q}_{0}+\mathbf{q}\right)
$$

\subsection{Quadratic ANCF element}

For the quadratic ANCF beam element, three nodes are chosen at the end points and midpoint of the beam axis, as depicted in Fig. 1(b). The displacements $\mathbf{u}^{(1)}, \mathbf{u}^{(2)}, \mathbf{u}^{(3)}$ and displacement gradients $\mathbf{u}_{, \eta}^{(1)}, \mathbf{u}_{, \eta}^{(2)}, \mathbf{u}_{, \eta}^{(3)}$ at the nodal points are chosen as degrees of freedom. Therefore, the unknowns for the additional node are added to the unknowns of the linear case in the following way:

$$
\mathbf{q}=\left[\begin{array}{llllll}
\mathbf{u}^{(1) T} & \mathbf{u}_{, \eta}^{(1) T} & \mathbf{u}^{(2) T} & \mathbf{u}_{, \eta}^{(2) T} & \mathbf{u}^{(3) T} & \mathbf{u}_{, \eta}^{(3) T}
\end{array}\right]^{T} .
$$

For the additional midpoint node, the initial position $\mathbf{r}_{0}^{(3)}=\left[q_{0}, q_{0}{ }_{10}\right]^{T}$ and the initial direction of the cross section $\mathbf{r}_{0, \eta}^{(3)}=\left[q_{0} 11, q_{0}{ }_{12}\right]^{T}$ are introduced, see Fig. 4 . For the nodes at 
the end points of the element, all notations from the linear ANCF element are kept. In the quadratic case, the displacement vector $\mathbf{u}$ is interpolated quadratically by $m=6$ shape functions, leading again to the relation in (6). The shape functions are chosen similar to those given in [9] as

$$
\begin{array}{ll}
S_{1}(\xi, \eta)=-\frac{2}{L^{2}} \xi\left(\frac{L}{2}-\xi\right), & S_{2}(\xi, \eta)=\eta S_{1}(\xi, \eta), \\
S_{3}(\xi, \eta)=+\frac{2}{L^{2}} \xi\left(\frac{L}{2}+\xi\right), & S_{4}(\xi, \eta)=\eta S_{3}(\xi, \eta), \\
S_{5}(\xi, \eta)=-\frac{4}{L^{2}}\left(\xi-\frac{L}{2}\right)\left(\xi+\frac{L}{2}\right), & S_{6}(\xi, \eta)=\eta S_{5}(\xi, \eta) .
\end{array}
$$

The shape function matrix $\mathbf{S}$ is built similarly to (8) for the linear ANCF beam element.

\section{Strain energy in case of ANCF elements}

\subsection{Standard continuum mechanics based formulation}

The original formulation for a shear deformable ANCF beam is based on the work of Omar and Shabana [10]. Herein, the energy of elastic forces follows from the continuum mechanics approach, using the relation between the nonlinear Green-Lagrange strain tensor and the second Piola-Kirchhoff stress tensor. The Green-Lagrange strain tensor $\mathbf{E}$ is defined as

$$
\mathbf{E}=\frac{1}{2}\left(\mathbf{F}^{T} \mathbf{F}-\mathbf{I}\right)
$$

in which the deformation gradient $\mathbf{F}$ is defined via the derivatives of the position in the following way:

$$
\mathbf{F}=\frac{\partial \mathbf{r}}{\partial \mathbf{r}_{0}}=\frac{\partial \mathbf{r}}{\partial \boldsymbol{\xi}} \frac{\partial \boldsymbol{\xi}}{\partial \mathbf{r}_{0}}=\left[\begin{array}{ll}
\frac{\partial r_{1}}{\partial \xi} & \frac{\partial r_{1}}{\partial \eta} \\
\frac{\partial r_{2}}{\partial \xi} & \frac{\partial r_{2}}{\partial \eta}
\end{array}\right]\left[\begin{array}{ll}
\frac{\partial r_{01}}{\partial \xi} & \frac{\partial r_{01}}{\partial \eta} \\
\frac{\partial r_{02}}{\partial \xi} & \frac{\partial r_{02}}{\partial \eta}
\end{array}\right]^{-1},
$$

in which the vector $\xi=(\xi, \eta)$ denotes the coordinates of the straight scaled reference element in unit configuration. The transformation between the scaled straight element and the possibly distorted reference element is included in the element Jacobian

$$
\mathbf{J}=\frac{\partial \mathbf{r}_{0}}{\partial \xi} .
$$

In case of a straight and undistorted reference configuration, the element Jacobian simply is $\mathbf{J}=\mathbf{I}$. For the Green-Lagrange strain $\mathbf{E}$ and the second Piola-Kirchhoff stress tensor, often denoted by $\mathbf{S}$ in literature, the engineering strain vector $\boldsymbol{\varepsilon}$ and stress vector $\boldsymbol{\sigma}$ are defined as

$$
\boldsymbol{\varepsilon}=\left[\begin{array}{lll}
E_{\xi \xi} & E_{\eta \eta} & 2 E_{\xi \eta}
\end{array}\right]^{T} \text { and } \quad \boldsymbol{\sigma}=\left[\begin{array}{lll}
S_{\xi \xi} & S_{\eta \eta} & S_{\xi \eta}
\end{array}\right]^{T} .
$$

In case of a plane state of stress, the constitutive relations are contained in the elasticity matrix

$$
\mathbf{D}=\frac{E}{\left(1-v^{2}\right)}\left[\begin{array}{ccc}
1 & v & 0 \\
v & 1 & 0 \\
0 & 0 & \frac{1-v}{2}
\end{array}\right]
$$


in which $E$ is Young's modulus and $v$ is Poisson's ratio. The stress-strain relation can now be written as

$$
\sigma=\mathbf{D} \boldsymbol{\varepsilon}
$$

In general, the strain energy of a beam element with a rectangular cross section is written in the following form:

$$
U_{\text {standard }}^{\mathrm{CM}}=\frac{1}{2} \int_{-H / 2}^{H / 2} \int_{-L / 2}^{L / 2} \boldsymbol{\varepsilon}^{T} \mathbf{D} \boldsymbol{\varepsilon} \operatorname{det}(\mathbf{J}) d \xi d \eta,
$$

in which the abbreviation $\mathrm{CM}$ indicates the continuum mechanics approach.

\subsection{Enhanced continuum mechanics based formulation}

The main problem of the standard continuum mechanics based formulation is the Poisson ratio $v$ which couples axial strains $E_{\xi \xi}$ and the transverse normal strains $E_{\eta \eta}$ in the stressstrain relation (17). Thus, an axial stretch of the unconstrained beam leads to a thickness deformation. This effect induces Poisson locking, which has to be avoided in order to obtain useful results also for thin beams. Gerstmayr et al. [3] suggested to split the elasticity matrix D into two parts,

$$
\mathbf{D}=\mathbf{D}^{0}+\mathbf{D}^{v}
$$

in which $\mathbf{D}^{0}$ does not include the Poisson ratio $v$, while $\mathbf{D}^{v}$ involves the Poisson effect only. In more detail, $\mathbf{D}^{0}$ includes the Young's modulus $E$ and the shear modulus $G$ in the diagonal;

$$
\mathbf{D}^{0}=\left[\begin{array}{ccc}
E & 0 & 0 \\
0 & E & 0 \\
0 & 0 & G k_{s}
\end{array}\right]
$$

The shear correction factor $k_{s}$ accounts for the distribution of the shear stress along the cross section. The Poisson effect in the axial and transverse deformation is captured in $\mathbf{D}^{v}$ as follows:

$$
\mathbf{D}^{v}=\frac{E}{\left(1-v^{2}\right)}\left[\begin{array}{ccc}
v^{2} & v & 0 \\
v & v^{2} & 0 \\
0 & 0 & 0
\end{array}\right]
$$

Applying (19), the strain energy in (18) is split into two parts,

$$
\begin{aligned}
U_{\mathrm{enh} .}^{\mathrm{CM}}= & \frac{1}{2} \int_{-H / 2}^{H / 2} \int_{-L / 2}^{L / 2} \boldsymbol{\varepsilon}^{T} \mathbf{D}^{0} \boldsymbol{\varepsilon} \operatorname{det}(\mathbf{J}) d \xi d \eta \\
& +\frac{1}{2} H \int_{-L / 2}^{L / 2} \boldsymbol{\varepsilon}^{T} \mathbf{D}^{v} \boldsymbol{\varepsilon} \operatorname{det}(\mathbf{J}) d \xi
\end{aligned}
$$

considering the Poisson effect only at the beam axis $\eta=0$. This enhanced formulation eliminates the Poisson locking effect. 


\subsection{Formulation based on Reissner, Simo and Vu-Quoc}

In contrast to the standard and the enhanced formulation, described in Sects. 4.1 and 4.2, in which the virtual work of elastic forces is deduced from the continuum mechanics approach, a different derivation for the strain energy in terms of the axial strain $\Gamma_{1}$, the shear strain $\Gamma_{2}$ and the bending strain $K$ is presented in the following. Reissner [11] stated the virtual work of the internal forces as

$$
\delta W=\int_{0}^{L}\left(M \delta K+N \delta \Gamma_{1}+Q \delta \Gamma_{2}\right) d s,
$$

in which the integration is performed over the reference coordinate $s$. The stress resultants, more precise the normal force $N$, the shear force $Q$ and the bending moment $M$ fulfill the generalized nonlinear constitutive relations

$$
N=f_{N}\left(\Gamma_{1}, \Gamma_{2}, K\right), \quad Q=f_{Q}\left(\Gamma_{1}, \Gamma_{2}, K\right), \quad M=f_{M}\left(\Gamma_{1}, \Gamma_{2}, K\right),
$$

in which the functions $f$ depend on a set of generalized strain measures, $\Gamma_{1}, \Gamma_{2}$ and $K$. In the present paper, the stress resultants and generalized strains are defined according to Simo and Vu-Quoc [14]. There, not only a linear relation between resultants and strain measures is assumed, but the different quantities are also decoupled, setting

$$
N=E A \Gamma_{1}, \quad Q=k_{s} G A \Gamma_{2}, \quad M=E I K .
$$

For the above definitions, the axial stiffness $E A$, the shear stiffness $G A$ with the shear correction factor $k_{s}=\frac{10(1+v)}{12+11 v}$ and the bending stiffness $E I$ are used. $E$ represents Young's modulus and $A$ is the area of the cross section. $G$ represents the shear modulus, $I$ is the second moment of area of the cross section. The quantity $K$ denotes the change of angle of the cross section relatively to the reference length:

$$
K=\frac{\mathbf{r}_{, \eta} \times \mathbf{r}_{, \eta}^{\prime}}{\left|\mathbf{r}_{, \eta}\right|^{2}}
$$

The generalized strains $\Gamma_{1}$ and $\Gamma_{2}$ are introduced as follows:

$$
\Gamma_{1}=\mathbf{t}_{1}^{T} \mathbf{r}^{\prime}-1, \quad \Gamma_{2}=\mathbf{t}_{2}^{T} \mathbf{r}^{\prime},
$$

in which the vectors $\mathbf{t}_{1}$ and $\mathbf{t}_{2}$ are defined as

$$
\mathbf{t}_{2}=\frac{\mathbf{r}_{, \eta}}{\left|\mathbf{r}_{, \eta}\right|}, \quad \mathbf{t}_{1}=\frac{\hat{\mathbf{r}}_{, \eta}}{\left|\mathbf{r}_{, \eta}\right|}=\frac{1}{\left|\mathbf{r}_{, \eta}\right|}\left[\begin{array}{ll}
r_{2, \eta} & -r_{1, \eta}
\end{array}\right],
$$

in which $\hat{\mathbf{r}}$ is the rotation of vector $\mathbf{r}$ by 90 degrees. The combination of (23) and (25) corresponds to the following strain energy:

$$
U^{\text {Reissner }}=\frac{1}{2} \int_{-L / 2}^{L / 2}\left(E A \Gamma_{1}^{2}+k_{s} G A \Gamma_{2}^{2}+E I K^{2}\right) d \xi .
$$

In order to enhance the classical strain energy in (29), an additional term accounting for the strain energy due to thickness deformation has to be introduced. Following Gerstmayr et 
al. [3], the additional thickness strain energy $U^{\text {thickness }}$ can be defined, in case of a rectangular cross section, by

$$
U^{\text {thickness }}=\frac{1}{2} \int_{-L / 2}^{L / 2} E A E_{\eta \eta}^{2} d \xi,
$$

in which the transverse component $E_{\eta \eta}=\frac{1}{2}\left(\mathbf{r}_{, \eta}^{T} \mathbf{r}_{, \eta}-1\right)$ of the Green-Lagrange strain is used. The quantity $E_{\eta \eta}$ is independent of the stress resultants $\Gamma_{1}, \Gamma_{2}$ and $K$ defined above, since it only depends on the length $\left|\mathbf{r}_{, \eta}\right|$ of the slope vector, while $\Gamma_{1}, \Gamma_{2}$ and $K$ can be expressed in terms of the normalized vectors $\mathbf{t}_{1}, \mathbf{t}_{2}$ and the direction of the beam axis $\mathbf{r}^{\prime}$.

The total strain energy then reads

$$
U=U^{\text {Reissner }}+U^{\text {thickness }} .
$$

Due to the choice of $E_{\eta \eta}$, axial and thickness deformations are decoupled. The thickness strain energy (30) stabilizes the element with respect to thickness deformation. In case of no external compressive or tensile forces acting in vertical direction, the element thickness is not changed at all and a reduced stiffness of the deformation of the cross section may be taken into account without changing the overall behavior of the element while reducing the eigenfrequency of thickness modes. The model introduced in (30) corresponds to a material with Poisson's ratio $v=0$.

While the variation of the strain energy due to Simo and Vu-Quoc, (29), is integrated numerically using a Gaussian quadrature rule, for the terms due to thickness variation, (30), a Lobatto integration formula is utilized, where the nodal points of the element are used as integration points. Since the shape functions are defined on the scaled straight rectangular element and the integration points on the unit interval $[-1,+1]$, the element transformation (1) has to be included.

\section{Numerical examples}

In order to show the validity of the proposed ANCF elements and to illustrate the performance, several static and dynamic examples are considered.

Both the linear and the quadratic ANCF elements have been implemented in the framework of the multibody and finite element research code HOTINT. ${ }^{1}$ For verification, numerical examples taken from [3] were investigated using the proposed elements with the Reissner-Simo-Vu-Quoc strain energy in Sect. 4.3.

As a first example, a cantilever beam under two different vertical tip loads leading either to small or large deformation is studied. The beam is discretized with different numbers of linear and quadratic ANCF elements. In addition, the effect of reduced integration on the convergence is studied for the quadratic ANCF element.

In a second analysis, the natural frequencies of a simply supported beam are computed and the corresponding eigenvectors are classified. Additionally, a convergence study for the eigenfrequencies depending on the number of finite elements is presented.

Furthermore, the different formulations, namely the standard and the enhanced continuum mechanics based formulation as well as the Reissner-Simo-Vu-Quoc formulation, described in Sect. 4, are compared. The error of the tip displacement of the mentioned cantilever beam and the eigenfrequencies of the simply supported beam are utilized as reference values.

\footnotetext{
${ }^{1}$ http://tmech.mechatronik.uni-linz.ac.at/staff/gerstmayr/hotint.html.
} 
Fig. 5 Cantilever beam under a vertical tip load $F$

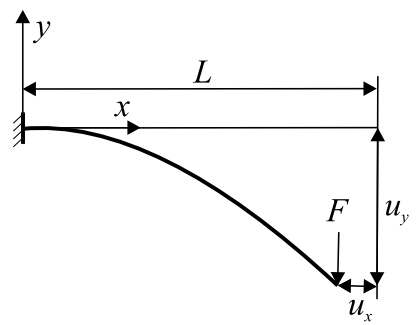

Table 1 Tip displacement in $x$ - and $y$-directions $u_{x}$ and $u_{y}$ of the cantilever beam in case of small deformations and absolute error for $u_{y}$ calculated with different numbers of linear and quadratic ANCF beam elements using the Reissner-Simo-Vu-Quoc formulation from Sect. 4.3

\begin{tabular}{llll}
\hline \# Elements & Displacement $u_{x}$ & Displacement $u_{y}$ & Abs. error for $u_{y}$ \\
\hline Linear ANCF beam element & & & \\
1 & $9.12273046 \mathrm{e}-8$ & $6.16666566 \mathrm{e}-4$ & 0.000193 \\
2 & $1.61293091 \mathrm{e}-7$ & $7.61594059 \mathrm{e}-4$ & $4.831 \mathrm{e}-5$ \\
4 & $1.81763233 \mathrm{e}-7$ & $7.97825954 \mathrm{e}-4$ & $1.208 \mathrm{e}-5$ \\
256 & $1.88847418 \mathrm{e}-7$ & $8.09900305 \mathrm{e}-4$ & $2.945 \mathrm{e}-9$ \\
Quadratic ANCF beam element & & & \\
1 & $1.86982122 \mathrm{e}-7$ & $8.09903209 \mathrm{e}-4$ & $4.1 \mathrm{e}-11$ \\
Simo-Vu-Quoc (MAPLE) [3] & $1.8884916 \mathrm{e}-7$ & $8.0990325 \mathrm{e}-4$ & \\
\hline
\end{tabular}

\subsection{Cantilever beam}

At first, a cantilever beam under a vertical tip load $F$ is studied. For a sketch of the problem setup, see Fig. 5. The beam has length $L=2 \mathrm{~m}$, cross section of height $h=0.5 \mathrm{~m}$ and width $w=0.1 \mathrm{~m}$. The beam is made of steel with a Young's modulus $E=2.07 \times 10^{11} \mathrm{~N} / \mathrm{m}^{2}$ and a Poisson ratio of $v=0.3$. The shear correction factor is chosen as $k_{s}=\frac{10(1+v)}{12+11 v}$. First, the case of small deformations is tested. To this end, the vertical tip load is set to $F=5 \times 10^{5} \cdot h^{3} \mathrm{~N}$.

In Table 1, the tip displacements for different discretizations of the cantilever beam are compared with the analytical solution of the equations from Simo and Vu-Quoc computed in Maple, which is taken from [3]. In this calculation, the linear as well as the quadratic ANCF beam elements use the Reissner-Simo-Vu-Quoc formulation. Moreover, the absolute error $\left\|u_{y}-u_{y}^{*}\right\|$ is provided, where $u_{y}^{*}$ denotes the analytically computed $y$-displacement. In the case of the linear ANCF beam element using 256 elements, the solution for the displacement in $y$-direction shows eight converged digits compared to the solution computed in MAPLE to an arbitrary precision. Using the quadratic ANCF beam element this level of accuracy is already obtained for a discretization using four elements. The quadratic ANCF beam element converges extremely fast with an agreement factor almost one using only one beam element.

For a vertical tip load $F=5 \times 10^{8} \cdot h^{3} \mathrm{~N}$, the cantilever beam undergoes large deformations. Again, the results are computed using the Reissner-Simo-Vu-Quoc formulation and are compared with the exact values provided in [3], see Table 3. Additionally, the fully parameterized ANCF element proposed in [3], which is originally based on the large rotation vector formulation by Simo and Vu-Quoc [14], is included in the comparison. The degrees 
Table 2 List of different elements, which are used to draw comparisons. As comparative values serve the number of nodes, the number of degrees of freedom and the induced convergence order for the tip displacements in the nodes

\begin{tabular}{llcl}
\hline Element & Nodes & DOF & Convergence order \\
\hline Fully parameterized ANCF element [3] & 2 & 12 & 2 \\
Linear ANCF beam element & 2 & 8 & 2 \\
Quadratic ANCF beam element & 3 & 12 & 4 \\
\hline
\end{tabular}

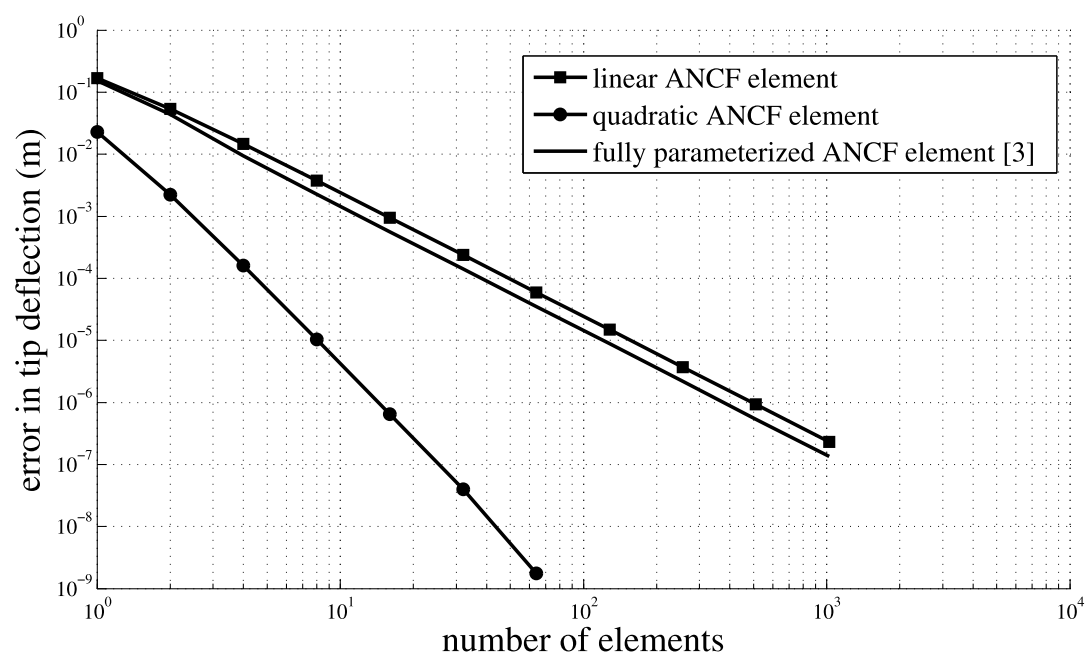

Fig. 6 Convergence plot for the cantilever beam example comparing the proposed linear and quadratic ANCF beam element using the Reissner-Simo-Vu-Quoc formulation from Sect. 4.3 and the fully parameterized ANCF element [3]

of freedom of this fully parameterized ANCF element [3] are position coordinates and two slope vectors in each of the two nodes. The element has twelve degrees of freedom. The position vector of the fully parameterized ANCF element [3] is of third order interpolation, while the rotation of the cross section is only interpolated linearly with respect to the beam axis. Even though one could expect a third order convergence for displacements in case of thin beams, the fully parameterized ANCF element [3] suffers from locking and therefore the convergence rate follows $\mathrm{O}\left(n^{2}\right)$, where $n$ is the number of elements, see Fig. 6 . The linear ANCF beam element consists of two nodes and eight degrees of freedom. Because it is not a fully-parameterized element, it needs less degrees of freedom compared to the fully parameterized ANCF element [3]. The convergence study in Fig. 6 demonstrates that the proposed linear ANCF element shows the same convergence order as the fully parameterized ANCF element [3]. The quadratic ANCF beam element consists of three nodes and therefore has twelve degrees of freedom. Even though the proposed quadratic ANCF element has the same number of degrees of freedom as the fully parameterized ANCF element [3], it shows essentially faster convergence, see Fig. 6. In Table 2, the numbers of nodes, degrees of freedom (DOF) and convergence orders for the three elements are listed.

For the linear and quadratic ANCF elements developed in this work, the variational equations of the Reissner-Simo-Vu-Quoc formulation are integrated numerically using Gaussian 
Table 3 Tip displacement in $x$-and $y$-directions $u_{x}$ and $u_{y}$ of the cantilever beam in case of large deformations and absolute error for $u_{y}$ calculated with variable numbers of linear and quadratic ANCF beam elements using the Reissner-Simo-Vu-Quoc formulation from Sect. 4.3 and compared to reference solution [3]

\begin{tabular}{|c|c|c|c|}
\hline \# Elements & Displacement $u_{x}$ & Displacement $u_{y}$ & Abs. error for $u_{y}$ \\
\hline \multicolumn{4}{|l|}{ Linear ANCF beam element } \\
\hline 1 & 0.07140274 & 0.54225823 & 0.168310 \\
\hline 2 & 0.12379212 & 0.65687111 & 0.053697 \\
\hline 4 & 0.14346767 & 0.69593561 & 0.014633 \\
\hline 8 & 0.14904162 & 0.70681526 & 0.003753 \\
\hline 16 & 0.15048522 & 0.70962389 & $9.447 e-4$ \\
\hline 1024 & 0.15097103 & 0.71056837 & $2.280 \mathrm{e}-7$ \\
\hline \multicolumn{4}{|l|}{ Quadratic ANCF beam element } \\
\hline 1 & 0.13971417 & 0.68775242 & 0.022816 \\
\hline 2 & 0.15005721 & 0.70833713 & 0.002231 \\
\hline 4 & 0.15090938 & 0.71040910 & 0.000159 \\
\hline 8 & 0.15096721 & 0.71055828 & $1.032 \mathrm{e}-5$ \\
\hline 16 & 0.15097090 & 0.71056795 & $6.480 \mathrm{e}-7$ \\
\hline \multicolumn{4}{|c|}{ Fully parameterized ANCF element [3] } \\
\hline 1 & 0.10088348 & 0.55810849 & 0.152460 \\
\hline 2 & 0.13526374 & 0.66661803 & 0.043950 \\
\hline 4 & 0.14764474 & 0.70117342 & 0.009395 \\
\hline 8 & 0.15016361 & 0.70829393 & 0.002275 \\
\hline 16 & 0.15077038 & 0.71000360 & $5.650 \mathrm{e}-4$ \\
\hline Simo-Vu-Quoc (MAPLE) [3] & 0.15097114 & 0.71056859 & \\
\hline
\end{tabular}

and Lobatto integration rules. If the terms are integrated exactly, locking occurs. A common way to avoid the locking phenomenon of finite elements is to use reduced integration. For the linear ANCF beam element, selective reduced integration is applied: the classical strain energy is integrated by a Gaussian integration rule of order one, while the thickness terms are integrated by a Lobatto integration rule of order two. For the quadratic ANCF beam element, third-order integration rules are used for all terms. The same integration schemes are applied to the standard and enhanced continuum mechanics formulation. Even though reduced integration is used, no hourglass modes appear.

In Table 4, the different formulations from Sect. 4 are compared. The tip displacements of the large deformation cantilever beam discretized with 2000 linear ANCF elements are compared. Due to Poisson locking, the standard continuum mechanics formulation does not show convergence to the analytical value computed in MAPLE given in Table 3. For a comparison of convergence of the Reissner-Simo-Vu-Quoc formulation and the standard continuum mechanics formulation with respect to the analytic value, see Fig. 7.

The following convergence study shows the accuracy of the proposed elements for the chosen integration schemes: the convergence rate of the quadratic ANCF beam element utilizing the Reissner-Simo-Vu-Quoc formulation with respect to the analytical solution is shown in Fig. 8 and the convergence rate of the quadratic ANCF beam element utilizing the enhanced continuum mechanics formulation with respect to the converged solution obtained with 2000 quadratic ANCF elements is shown in Fig. 9. The error in the tip displacement 
Table 4 Tip displacement in $x$ - and $y$-directions $u_{x}$ and $u_{y}$ of the different formulations using linear ANCF beam elements

\begin{tabular}{lll}
\hline Formulation & Displacement $u_{x}$ & Displacement $u_{y}$ \\
\hline 2000 linear ANCF beam elements based on & & \\
standard continuum mechanics form & 0.1350387 & 0.6626850 \\
enhanced continuum mechanics form & 0.1581778 & 0.7152418 \\
Reissner-Simo-Vu-Quoc & 0.1509710 & 0.7105683 \\
\hline
\end{tabular}

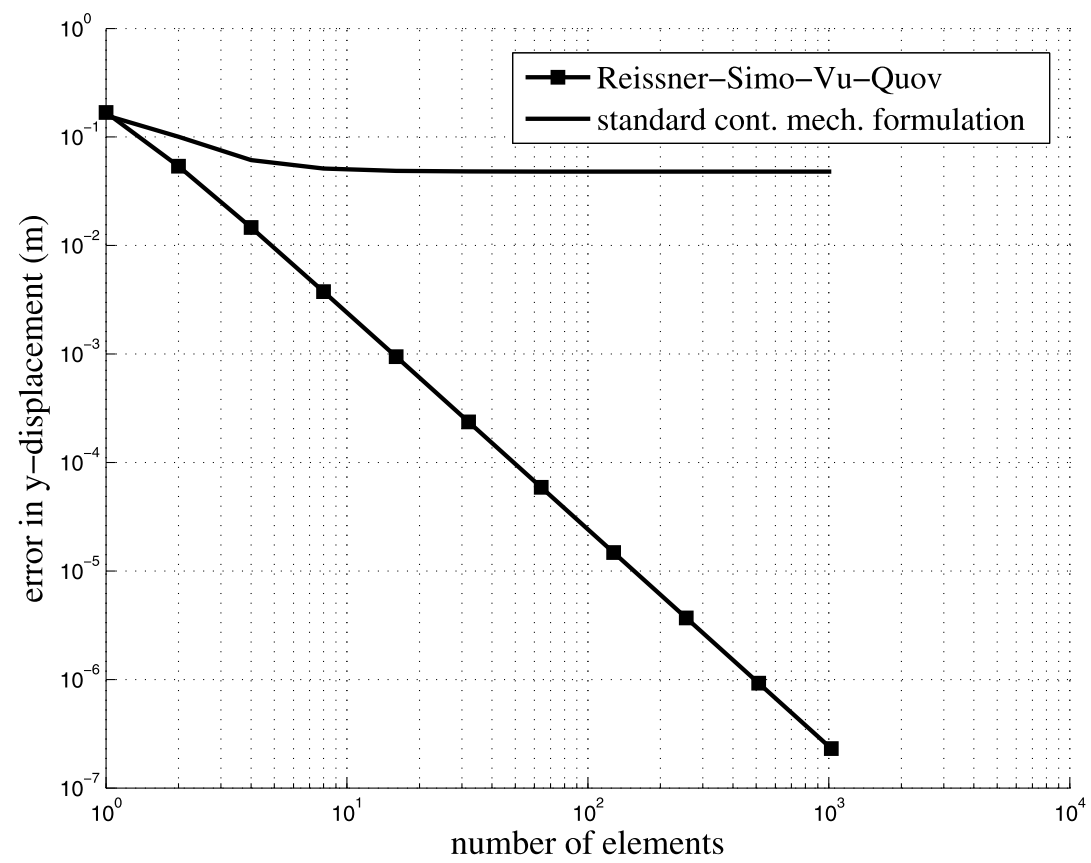

Fig. 7 Comparison of convergence of the Reissner-Simo-Vu-Quoc formulation and the standard continuum mechanics formulation with respect to the analytic solution calculated in MAPLE [3]

in $y$-direction is taken as a measure for the comparison of the accuracy of the solution with and without reduced integration order. In case of the linear ANCF beam element, the displacement at an arbitrary point converges linearly. In contrast, the convergence of the displacement at a nodal point is of quadratic order like in the case of the fully parameterized ANCF element [3], see Fig. 6. Similar effects were found for the quadratic ANCF beam element in the provided examples. Herein, the convergence rate of the nodal displacement follows $\mathrm{O}\left(n^{4}\right)$, see Fig. 8 for the Reissner-Simo-Vu-Quoc formulation and Fig. 9 for the enhanced continuum mechanics formulation. Since the convergence orders of the quadratic ANCF beam element utilizing either the Reissner-Simo-Vu-Quoc formulation or the enhanced continuum mechanics formulation are the same, the decision, which formulation should be chosen for an application problem, depends only on the material model. 


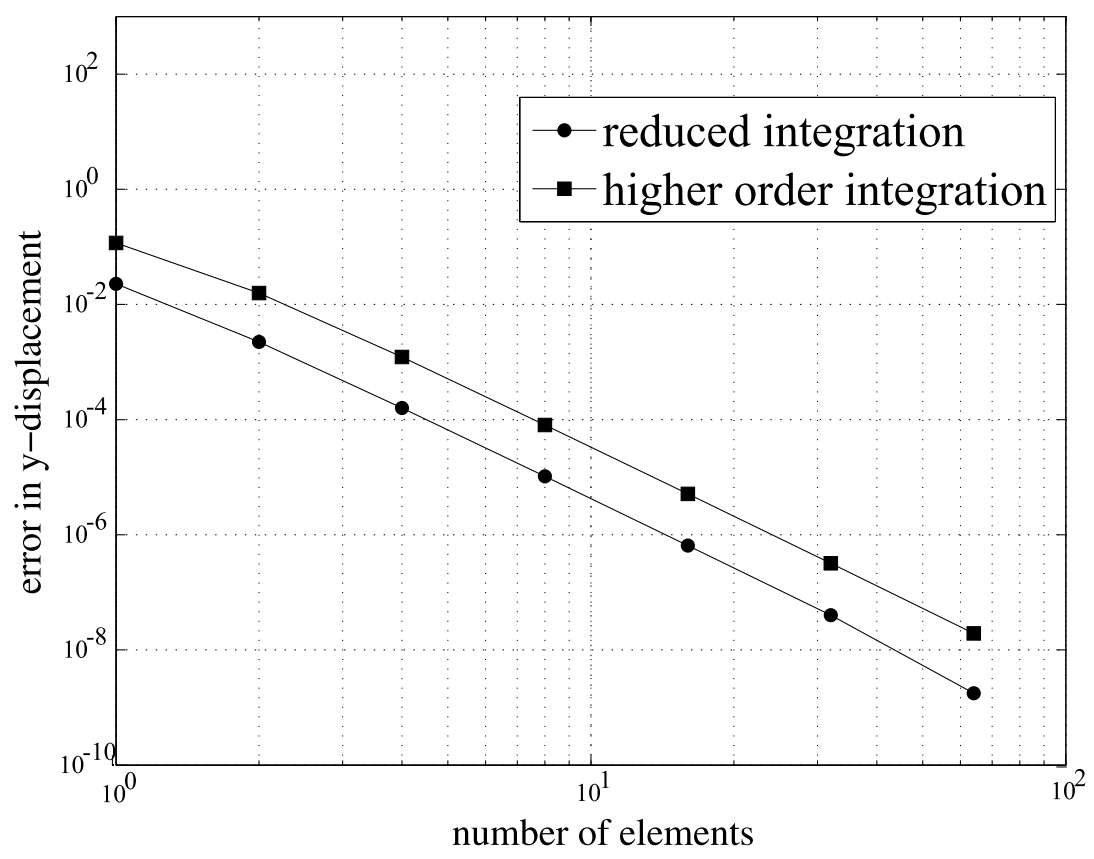

Fig. 8 Convergence of the quadratic ANCF beam element utilizing the Reissner-Simo-Vu-Quoc formulation compared to the analytical solution with and without reduced integration application

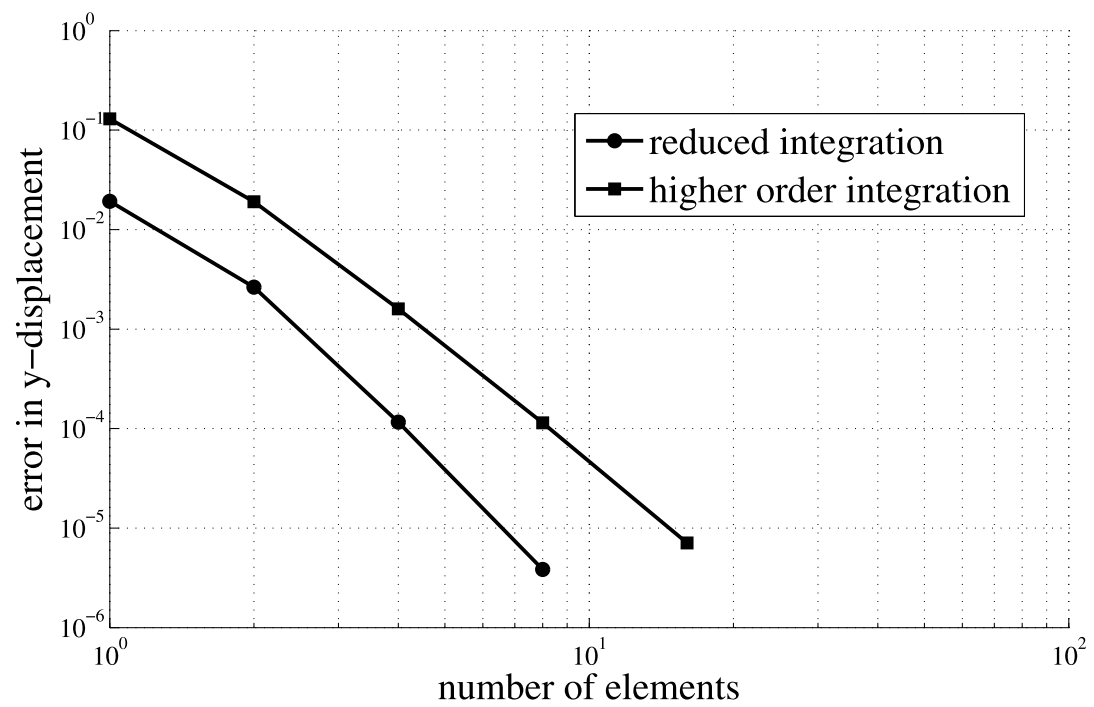

Fig. 9 Convergence of the quadratic ANCF beam element utilizing the enhanced continuum mechanics formulation compared to the converged solution with and without reduced integration application 


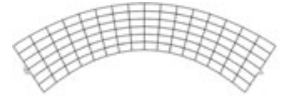

Mode 1: 105.15

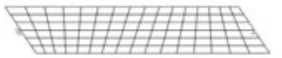

Mode 4: 1382.33

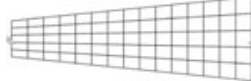

Mode 7: 3090.98

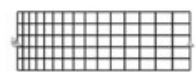

Mode 2: 281.37

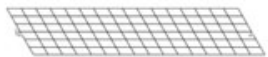

Mode 5: 1766.99



Mode 8: 3090.98

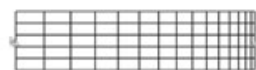

Mode 3: 1012.36

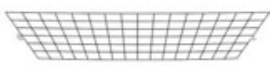

Mode 6: 1896.70

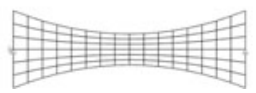

Mode 9: 4887.26

Fig. 10 Eigenfrequencies $(\mathrm{rad} / \mathrm{s})$ and corresponding mode shapes

\subsection{Eigenfrequencies of a simply supported beam}

In this section, the eigenfrequencies of a simply supported beam are studied. Since analytic solutions for the eigenfrequencies exist for this case, the following beam parameters in accordance with [3] are utilized: length $L=2 \mathrm{~m}$, height $h=0.4 \mathrm{~m}$, width $w=0.4 \mathrm{~m}$, density $\rho=7850 \mathrm{~kg} / \mathrm{m}^{3}$, Young's modulus $E=10^{9} \mathrm{~N} / \mathrm{m}^{2}$, Poisson ratio and shear correction factor remain unchanged with $v=0.3$ and $k_{s}=\frac{10(1+v)}{12+11 v}$.

In a further study, not shown in the results, it has been verified that three rigid body modes result from an eigenfrequencies analysis of a free-free beam.

To realize the bearing on the left hand side of the simply supported beam the displacements of the axis in $x$ - and $y$-directions are set to zero, for the slide bearing on the right only the displacement in $y$-direction is fixed. In the succeeding computations, the ReissnerSimo-Vu-Quoc formulation is utilized for a single quadratic ANCF beam element with twelve degrees of freedom. Since three degrees of freedom are eliminated by the boundary constraints, nine eigenvalues can be computed. See Fig. 10 for a visualization of the computed eigenmodes. In addition, a convergence analysis for the eigenfrequencies depending on the number of elements is presented for the quadratic ANCF beam element. Reduced integration is used in order to obtain fast convergence. As a consequence, the eigenfrequencies are lower than in the computation without reduced integration. The computed values are compared to the values from Table 6 in [3]. Here, Simo-Vu-Quoc $\theta$-quad, r-quad denotes the eigenfrequencies of the original second order element by Simo and Vu-Quoc, while Timoshenko (anal.) represents the analytical solution. As a result of quadratic interpolation order, the eigenfrequencies converge quickly to the analytic solution, see Table 5 . The convergence analysis of the first bending mode shows a convergence order of $O\left(n^{4}\right)$. The numerical values of shear and thickness eigenfrequencies calculated with only one quadratic ANCF element agree with the values for the Simo and Vu-Quoc beam in [3]. The quadratic ANCF element has additional thickness modes as compared to [3]. If relevant to an application, this seams to be the only disadvantage of the proposed element. Even though the thickness modes seem to be very large, the factor between the shear mode (mode 5) and the two smaller thickness modes (modes 7 and 8 ) is merely about 1.75 . However, to reveal the disadvantage of the quadratic ANCF beam element, the third thickness mode (mode 9) is nearly 2.8 times as large as the pure shear mode. Thus, the present elements can be applied similarly to classical nonlinear rod elements and for stiff problems time integrators should be applied to dynamic problems. 
Table 5 Numerical values of bending and axial eigenfrequencies ( $\mathrm{rad} / \mathrm{s})$ with a different number of elements utilizing the Reissner-Simo-Vu-Quoc formulation and comparison to analytical values

\begin{tabular}{lllll}
\hline \# Elements & \multicolumn{3}{l}{ Modes } & \\
\cline { 3 - 5 } & First bend. & First axial & 2nd bend. & 2nd axial \\
\hline Quadratic ANCF beam element & & & & \\
1 & 105.148 & 281.373 & $1382.33^{\mathrm{a}}$ & 1012.36 \\
2 & 96.642 & 280.392 & 358.979 & 854.85 \\
4 & 95.702 & 280.325 & 335.218 & 842.012 \\
8 & 95.638 & 280.321 & 332.436 & 841.031 \\
16 & 95.634 & 280.321 & 332.247 & 840.966 \\
Simo-Vu-Quoc $\theta$-quad, $r$-quad & 105.148 & 281.372 & 2243.456 & 1012.35 \\
Timoshenko (anal.) & 95.634 & 280.321 & 332.235 & 840.962 \\
\hline
\end{tabular}

${ }^{a}$ Computing the eigenfrequencies without using reduced integration leads to the same value as the Simo-VuQuoc reference value 2243.456.

Table 6 Numerical values of eigenfrequencies ( $\mathrm{rad} / \mathrm{s}$ ) with one linear ANCF beam element classified in the different mode shapes: axial, bending, shear and thickness modes

\begin{tabular}{llllll}
\hline Formulation & Modes & & & & \\
\cline { 2 - 6 } & Axial & Bending & Shear & Thickness & Thickness \\
\hline $\begin{array}{l}\text { One linear ANCF element based on } \\
\text { standard continuum mechanics form. }\end{array}$ & 308.957 & 2023.52 & 1916.94 & 3241.7 & 3262.83 \\
enhanced continuum mechanics form. & 308.957 & 1872.01 & 1766.99 & 3241.7 & 3259.44 \\
Reissner-Simo-Vu-Quoc & 309.098 & 618.195 & 1766.99 & 3090.98 & 5353.73 \\
\hline
\end{tabular}

Table 7 Numerical values of eigenfrequencies ( $\mathrm{rad} / \mathrm{s})$ with one quadratic ANCF beam element

\begin{tabular}{|c|c|c|c|c|}
\hline \multirow[t]{2}{*}{ Formulation } & \multicolumn{4}{|l|}{ Modes } \\
\hline & First bend. & First axial & 2nd bend. & 2nd axial \\
\hline \multicolumn{5}{|l|}{ One quadratic ANCF element based on } \\
\hline standard continuum mechanics form. & 110.606 & 281.304 & 1449.07 & 1007.67 \\
\hline enhanced continuum mechanics form. & 105.149 & 281.298 & 1382.33 & 1007.57 \\
\hline Reissner-Simo-Vu-Quoc & 105.148 & 281.373 & 1382.33 & 1012.36 \\
\hline
\end{tabular}

In addition to the convergence study of the eigenvalues for the quadratic ANCF beam element, an analysis of eigenmodes for a single linear and a single quadratic ANCF beam element is presented in order to compare Reissner-Simo-Vu-Quoc formulation with the standard and the enhanced continuum mechanics based formulation, mentioned in Sect. 4. In Table 6 all eigenfrequencies for a linear ANCF beam element are classified in different mode shapes. In Table 7, the first two bending and axial eigenfrequencies for a quadratic ANCF beam element employing the different formulations are presented. 


\section{Conclusion}

In the current paper, linear and quadratic shear deformable beam elements with bending, axial and shear deformation properties are presented. The ANCF finite elements are designed for large deformation and multibody dynamics problems. The deformation energy according to Simo and Vu-Quoc [14] is employed to the proposed elements, enhanced by a term accounting for thickness deformation and locking is eliminated.

In numerical examples it is shown that the standard continuum mechanics formulation suffers from locking, while the Reissner-Simo-Vu-Quoc and the enhanced continuum mechanics formulation are locking-free and have same order of convergence. The results of the proposed elements converge to the results in [3]. The decision, which formulation should be used for an application problem, is dependent on the utilized material law.

It has to be emphasized, that the previously published fully parameterized ANCF element [3] has 50\% more degrees of freedom as compared to the proposed linear element, while both elements have the same order of convergence. The proposed quadratic ANCF element has the same number of degrees of freedom as the original ANCF element [3], however it shows fourth order convergence instead of second order convergence in the original ANCF element.

Thus, in comparison to previously published elements, the proposed linear element is much more efficient, because it needs only one integration point for the integration of the elastic forces compared to eight or ten integration points which are necessary in the original ANCF element.

The proposed quadratic element has still less integration points compared to the original ANCF element, while the convergence is much faster.

Acknowledgements K. Nachbagauer and A.S. Pechstein acknowledge support from the Austrian Science Funds FWF via the project I337-N18, J. Gerstmayr and H. Irschik from the K2-Comet Austrian Center of Competence in Mechatronics ACCM.

Open Access This article is distributed under the terms of the Creative Commons Attribution Noncommercial License which permits any noncommercial use, distribution, and reproduction in any medium, provided the original author(s) and source are credited.

\section{References}

1. Gams, M., Planinc, I., Saje, M.: The strain-based beam finite elements in multibody dynamics. J. Sound Vib. 305, 194-210 (2007)

2. Gerstmayr, J., Irschik, H.: On the correct representation of bending and axial deformation in the absolute nodal coordinate formulation with an elastic line approach. J. Sound Vib. 318, 461-487 (2008)

3. Gerstmayr, J., Matikainen, M.K., Mikkola, A.M.: A geometrically exact beam element based on the absolute nodal coordinate formulation. J. Multibody Syst. Dyn. 20, 359-384 (2008)

4. Gerstmayr, J., Shabana, A.A.: Analysis of thin beams and cables using the absolute nodal coordinate formulation. Nonlinear Dyn. 45(1-2), 109-130 (2006)

5. Irschik, H., Gerstmayr, J.: A continuum mechanics based derivation of Reissner's large-displacement finite-strain beam theory: the case of plane deformations of originally straight Bernoulli-Euler beams. Acta Mech. 206, 1-21 (2009)

6. Irschik, H., Gerstmayr, J.: A hyperelastic Reissner-type model for non-linear shear deformable beams. In: Troch, I., Breitenecker, F. (eds.) Proceedings of the Mathmod 09 Vienna (2009)

7. Kerkkänen, K.S., Sopanen, J.T., Mikkola, A.M.: A linear beam finite element based on the absolute nodal coordinate formulation. ASME J. Mech. Des. 127, 621-630 (2005)

8. Matikainen, M.K., von Hertzen, R., Mikkola, A.M., Gerstmayr, J.: Elimination of high frequencies in the absolute nodal coordinate formulation. In: Proceedings of the Institution of Mechanical Engineers, Part K, Journal of Multibody Dynamics (2009) 
9. Mikkola, A.M., Garcia-Vallejo, D., Escalona, J.L.: A new locking-free shear deformable finite element based on absolute nodal coordinates. Nonlinear Dyn. 50, 249-264 (2007)

10. Omar, M.A., Shabana, A.A.: A two-dimensional shear deformable beam for large rotation and deformation problems. J. Sound Vib. 243(3), 565-576 (2001)

11. Reissner, E.: On one-dimensional finite-strain beam theory: the plane problem. J. Appl. Math. Phys. 23, 795-804 (1972)

12. Shabana, A.A.: Definition of the slopes and the finite element absolute nodal coordinate formulation. Multibody Syst. Dyn. 1(3), 339-348 (1997)

13. Shabana, A.A.: Dynamics of multibody systems (3rd edn). Cambridge University Press, New York (2005)

14. Simo, J.C., Vu-Quoc, L.: On the dynamics of flexible beams under large overall motions-the plane case: Part I and II. J. Appl. Math. 53, 849-863 (1986)

15. Sopanen, J.T., Mikkola, A.M.: Description of elastic forces in absolute nodal coordinate formulation. Nonlinear Dyn. 34, 53-74 (2003)

16. Yakoub, R.Y., Shabana, A.A.: Three-dimensional absolute nodal coordinate formulation for beam elements. ASME J. Mech. Des. 123, 606-621 (2001) 